\title{
AusTalk - The Australian Speech Database: Design Framework, Recording Experience and Localisation
}

\author{
Sharifa Alghowinem ${ }^{1,3}$, Michael Wagner ${ }^{2,1}$, Roland Goecke ${ }^{2,1}$ \\ ${ }^{1}$ Australian National University, Canberra, Australia, \\ ${ }^{2}$ University of Canberra, Canberra, Australia, \\ ${ }^{3}$ Ministry of Higher Education: Kingdom of Saudi Arabia, \\ sharifa.alghowinem@anu.edu.au,michael.wagner@canberra.edu.au, roland.goecke@ieee.org
}

\begin{abstract}
Aiming to create a comprehensive Australian speech database, the "AusTalk" project was carefully designed by 30 speech scientists contributing their disciplinary expertise. Standardised three one-hour audio-visual sessions for each of 1000 speakers around Australia were recorded having diverse components suitable for different research areas. The design of this database provides a good framework for any speech data corpus collection. In this paper, we present the AusTalk design and recording protocol, as well as problems faced and lessons learned. Localisation of this protocol and the potential customisation based on other countries' specifications are discussed. Collecting such speech databases including accent groups is encouraged to boost speech research in areas such as linguistics, speech and speaker recognition, forensic voice comparison, auditory-visual speech processing and many more.
\end{abstract}

Keywords-Speech corpus; audio-visual data; Australian English; generalisation;

\section{INTRODUCTION}

Given the rapid development in speech science and technology, a comprehensive speech database is needed to scale up the usage of related technologies and the findings of related research areas. Speech studies are not limited any longer to the study of speech production, but they also cover diverse research areas including theoretical and technical ones.

Theoretical speech research areas include the history of changes of language structure and phonetics over time, psychological (e.g. emotions) and physical (e.g. speech disorders) influences on speech, and social interactions. Recording the current state of the language will enable long-term comparison with future changes, as well as comparisons with older databases if they exist. Recognising emotions in speech is a common trend that would help developing a better understanding of the emotional (e.g. anger [1]) and mental state (e.g. depression [2]), which could be used for several applications. Recognising physical speech disorders and their production might help improve their treatments [3], [4]. However, speech and language history, psychology, and sociology differ based on cultures and geographical location, which demands different speech databases for each culture and language.

A wide range of technical research areas are involved with speech, which have a huge effect on business and security. Organisations are increasingly interested in automating customer services by supporting the development of human-computer interaction (HCI) technology. One of the technical areas of speech research that hugely contributes to other technologies is automatic speech recognition (ASR), which converts speech into text. The text can then be used for various HCI systems. The ASR systems obviously are language-dependent and are also influenced by the accent. Automated call centres, which already can be used in some booking systems, are one example of ASR applications. Such auto-answering interaction systems have recently become concerned about the emotional state of the customer as well. That is, to be sure that the customer is not annoyed or frustrated [5], [6], [1]. Other automated HCI systems such as virtual avatars answering basic visiting customers' questions have also been implemented lately. The Thinking Head is an example of such systems, which embodies human attributes and improves human-machine interaction [7].

Such interaction systems need to be trained on a database containing the language and accents of the potential customers to perform reasonably well. For example, the performance of an auto-phone-answering system trained with native speakers only is weak when used to interact with accented customers (e.g. foreigners) [8]. Speaker recognition and authentication are other technical areas that need a specified background model, which consists of speech samples of the general related population. A background model is used in such systems to check if a speakers characteristics are typical for the general population [9]. A background model requires several specifications, such as having a sufficient number of speech samples and speakers that are selected based on characteristics of the speaker in question. That is, if the target speaker is a male with accented speech, the background model should preferably contain accented male speakers without including the target speaker.

Speaker recognition systems are also used in forensic science to analyse the voice of a suspect. Scaling up such systems to recognise international criminals is a logical next step, which needs a worldwide collaboration to collect larger databases including several languages and accents. Collecting such speech databases including accent groups will encourage and boost speech research, especially in the forensic area. Therefore, the demand is increasing to create accent specific speech databases to be used in such studies and technologies.

Speech databases that could cover most of those research areas should be carefully designed to meet the expectations. Reducing variability in content and in recording environment by standardising procedure and recording equipment are key issues in such database collections. In Australia, 30 speech scientists from 13 institutions have met to contribute their disciplinary expertise and to optimally design an Australian 
speech database collection project. This project, formerly named "Big Australian Speech Corpus (Big ASC)", is now known to the public as "AusTalk".

In this paper, we describe the framework used in "AusTalk" as a comprehensive speech database that could be exemplary for any multi-disciplinary speech data collection, as well as problems faced and lessons learned from the recording process. We suggest the localisation of this protocol and the potential customisation depending on a country's specifications.

\section{Austalk Design}

\section{A. Motivation}

Several aspects motivated the design of the AusTalk project, where the rapid advancement in speech science and its applications inspired plans for a new extensive Australian speech database:

- $\quad$ Given the distinctive Australian English compared to American and British English, a specific Australian English speech database is needed for all speech technology applications. Those previous examples will be improved by using a specified Australian English speech database.

- The only Australian English speech database is ANDOSL, which was recorded in 1993-1995 [10] and the samples collected were too few given the current state of speech research that demands a larger database. ANDOSL is an audio-only database with just a single session per speaker from 108 speakers in total. Besides, ANDOSL suffers from a limited bandwidth, a lack of disordered speech, and limited coverage of indigenous speech and ethno-cultural Australian English variation. Therefore, ANDOSL has a limited use for Australian speech technology and applications such as ASR or speaker verification.

- Moreover, given the nature of Australia being multicultural due the diversity of immigrants, almost $20 \%$ of Australians are bilingual [11], which had its influence on Australian English. With the increase of immigration, there will be more changes in the language structure and accent in the future. Therefore, preserving the current state of Australian English is an urgent need to study and compare the phonetics and linguistics influenced by such changes from the past to the future. Also, such preservation has to take into account the variation of Australian English over geographical regions as well as ethno-cultural and social backgrounds.

- Another need for a new Australian speech database is recording and collecting speech samples for indigenous Australian languages. An estimate of the number of indigenous languages spoken in Australia in the 18th century was about 250 [12]. Unfortunately, $90 \%$ of the number of indigenous languages spoken fluently in Australia have disappeared since 1800 [13]. Therefore, preserving aboriginal languages is a key goal for such a database.
- Speech pathology studies are concerned with understanding speech disorders in order to develop intervention strategies and devices [3], [14], [15]. Having an Australian English speech database that has both typical and disordered speech will deepen such understanding for Australia accents.

Knowing that some of these motivations and goals were implemented and collected on a small scale at a few sites as a test, the next phases of AusTalk will expand on those goals. For example, indigenous Australian languages were recorded in a few recording sites, as well as emotional and disordered speech. More specified collection protocols for these examples will be implemented in the next phases.

\section{B. Participants}

The goal of AusTalk is to have a comprehensive and diverse speech sample to be able to cover most of the research interests. In order to accomplish that, a large database including regional and ethno-cultural coverage, as well as age, gender, and educational background variations is needed. In AusTalk, 1000 adult speakers participate in the data collection, with equal gender balance, and equal age groups, where participants were divided into three groups $(<25,30-45,>50)$, as well as having a balance of two socioeconomic-level groups. A webbased registration site was advertised to the public to register their interest. A selection was performed on each site based on the number of participants needed for that site, and having an equal gender and age group balance.

The data collection took place in 17 sites around Australia, including regional areas. Moreover, speech data from the 4 largest ethno-cultural groups of Australia born citizens with parents from non-English speaking countries - Italian, Greek , Chinese and Lebanese [11] - was recorded, as well as indigenous, disordered and emotional speech.

\section{Recording Environment}

Ideally controlled recording environment would be preferable to get high quality and clean recording. However, finding such recording environment for all locations including in the field is not feasible. Therefore, a reasonably quiet room with good lighting might be sufficient. In the AusTalk data collection procedure, 1 minute of silent recording is required to normalize the speech against the overall noise. For AusTalk the room size has to be at least $3 \mathrm{~m} \times 3 \mathrm{~m}$ in order for the unfolded Black Box (see section II-D) to fit. Moreover, noise and light level are measured, not to exceed a certain threshold.

\section{Hardware}

Having standardized recording equipment is essential to reduce recording variability and to have comparable speech samples. Therefore, in AusTalk, 12 identical portable, selfcontained and cost-effective recording stations, called Black Boxex were made available to each recording site (see Figure 1). The equipment selected to build the Black Box has efficient specifications to guarantee high quality recording, and at the same time they were off-the-shelf elements to make it economical and easily replicable. In order to facilitate the recordings in regional sites, the Black Box was made portable 


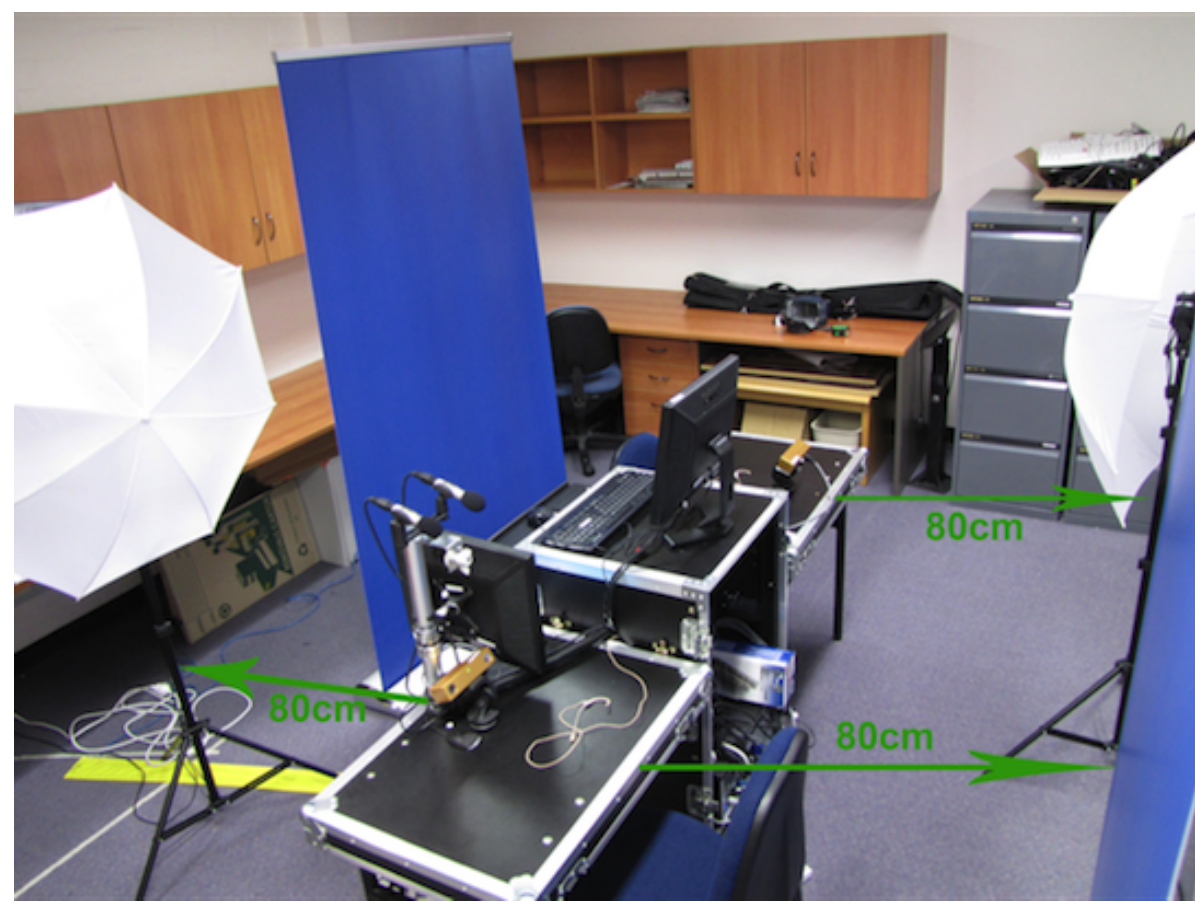

(a) Unfolded Black Box

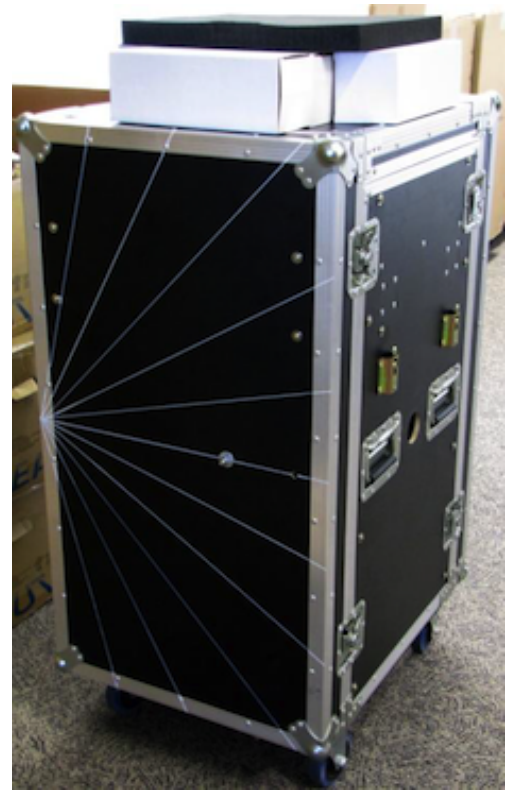

(b) Folded Black Box

Fig. 1. The AusTalk Recording Station

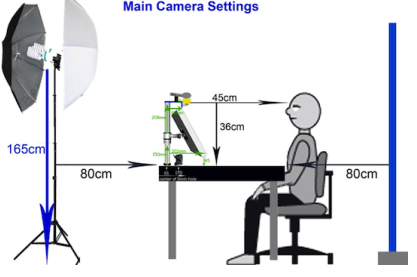

Fig. 2. The AusTalk Main Camera Setting and Distances

by packing it in a Mixer Rack Workstation (see Figure 1) that could be unpacked into 2 tables and a computer rack (see Figure 1). In its folded state, the Black Box dimensions are $550 \mathrm{~mm} \times 700 \mathrm{~mm} \times 1125 \mathrm{~mm}$, while unfolded its size will be $2475 \mathrm{~mm} \times 700 \mathrm{~mm} \times 1300 \mathrm{~mm}$, and it weighs $80 \mathrm{~kg}$. The Black Box is equipped with an audio recording device where the user could adjust sound volume levels, those levels were standardized over all the 13 recording sites. Moreover, the Black Box is equipped with 4 lights and 2 light mounts (see Figure 1) to ensure similar light conditions among the sites, with a few spare lights in case of miss-functional ones. In AusTalk settings, there are 2 internal hard-disks and another at least one external hard-drive for storing and backing-up the recordings, to be sent to the main storing servers in case of limited access to internet connection. For a list of equipment used in the Black Box refer to [16].

In General the Black Box recording station should have similar settings in each recording site as in Figure 2. Having similar distance between microphones, lights, and cameras will ensure stability and reduce variability between sessions, and sites.

\section{E. Software}

Automating the procedure of the recordings, reduces the risk of human errors. The software in AusTalk, has several functions including:

- Friendly interface to manage recording, including calibration prompts.

- Running recording sessions in sequential items and sections.

- Automating data acquisition by starting, stopping, and synchronizing hardware.

- Monitoring and checking data quality.

- Synchronising the audio and video devices

- $\quad$ Storing and labeling recording files

- Meta-data collection, storing and handling.

- Data compression and uploading and/or back-up data.

The AusTalk software, starts with the silent-recording, followed by adjusting volume levels and light. The research assistant (RA) then chooses the user ID from a drop-down list and asks to confirm the stored data (DoB) with the participant in order to start recording the session. The session sections and items are sequentially shown to the participant and controlled by the RA to move to the next item. By the end of each item, the software monitors and checks the data of that item for any errors, then stores it as raw data on the internal harddisk. Once the session finished, the software adds meta-data about the session, listing the items recorded and collecting some information about them, such as the name, time-stamps, 
2013 8th International Conference on Information Technology in Asia (CITA)

TABle I. Austalk StANDARd Protocol for EACH of The Three SEssions AND The Estimated Duration Time

\begin{tabular}{|c|c|c|c|c|c|}
\hline \multicolumn{2}{|l|}{ Session 1} & \multicolumn{2}{|l|}{ Session 2} & \multicolumn{2}{|l|}{ Session 3} \\
\hline Task & Time & Task & Time & Task & Time \\
\hline $\begin{array}{l}\text { Demographic, consent, } \\
\text { questionnaire }\end{array}$ & - & - & & - & \\
\hline $\begin{array}{c}\text { Calibration } \\
(+3 \mathrm{D} \text { face })\end{array}$ & 10 & Calibration & 3 & Calibration & 3 \\
\hline Opening Yes/No & 3 & Opening Yes/No & 2 & Opening Yes/No & 2 \\
\hline Words & 10 & Words & 10 & Words & 10 \\
\hline Read Story & 5 & Interview & 15 & Map Task (First run) & 20 \\
\hline Re-told Story & 10 & & & & \\
\hline Read Digits & 5 & Read Digits & 5 & Map Task (Second run) & 20 \\
\hline & & Read Sentences & 8 & Conversation & 5 \\
\hline & & & & Words & 10 \\
\hline Closing Yes/No & 2 & Closing Yes/No & 2 & Closing Yes/No & 2 \\
\hline Total & 44 & Total & 45 & Total & 72 \\
\hline
\end{tabular}

stored path and size. Those meta-data are useful for search ability and web server usability. By the end of each recording day, the RA is required to run the compression and uploading tool. In case Internet access is limited, the RA is supposed to back-up the compressed sessions into the external hard-drive to be sent to the recording center.

\section{F. Storage}

One of the main goals of AusTalk is to centralize the database to facilitate the access to it for all research centers. To accomplish that, a dedicated server with a friendly webinterface was built. The data compression mentioned earlier, served for the upload/download from the server purpose by minimizing the files size with minimum raw-data loss. The meta-data recorded from each session is pulled out to construct the web-interface to show each item's information and downloading path. Given the huge data collection process in AusTalk, and the great demand on the server bandwidth, certain specifications were taken into account while building the server to assure the server availability. Appropriate disk storage capacity is used not only for the recordings, but also for documentations, software and back-ups. Sufficient bandwidth is also allocated for this server, to manage and facilitate the data transfers and access between research centers.

\section{G. Protocol}

The comprehensive protocol of AusTalk was designed to cover the needs of both theoretical and technical speech research areas. The general design of AusTalk protocol contain 3 sessions, one hour each per participant. Those three sessions are recorded in three separate occasions (at least one week apart), to capture within-speaker variability. Table I lists the components of each session, which are detailed as follows:

1) Demographics: A comprehensive survey was filled by each participant in the first session. The survey covers demographics, family, and historical data. Each participant is asked about all regions and cities that they ever lived in, as well as several questions about the participant's parents and their ethno-cultural background, to investigate the influence in the participant accent. Educational and employment history, languages spoken, and speech and hearing proficiency (e.g., if they have singing or musical training, or if they have a hearing disability, etc.), are questioned in the survey to cover most of the participant's proficiency level and background that might affect their speech style.
2) Calibration: Beside the 1-min silent-recording mentioned earlier to calibrate the room noise (e.g., air-conditioning, workstations, any external noise), calibrating speakers speech and audio is performed in the beginning of each session, where the participant is asked to read isolated vowels. In the first session, the participant is asked to face the camera from different angles, in order to create a 3D face model for each speaker.

3) Digits: A simple isolated digits reading task was included in AusTalk protocol design. The specific reason of this task is to provide data for background database for audio password systems. Sequences were selected to ensure each of the basic 10 digits occurs at least once, to capture any co-articulatory variations and to provide sufficient acousticphonetic variation.

4) Reading Individual Words Task: A total of 322 words were selected in this task to be read by the speakers. Those words were selected to cover three word types: (1) monosyllabic words comprising stressed vowels of Australian English, (2) additional words addressing specific Australian English characteristics, and (3) polysyllabic words sampling stress patterns and variations [17]. Those words are beneficial for vowel examination, forensic work and socio-phonetic variations between speakers, as well as for comparison with similar databases.

5) Read Sentences: A set of 58 sentences is designed to elicit connected speech in a standard format. Those sentences sample all vowels and consonants in a connected speech, which will allow direct comparison of various connected Australian English speech from AusTalk or other databases.

6) Read Story: A story was chosen to be read by the speakers, which is another type of the connected speech style. Unlike the read sentences, participants where asked to read the story with emotional affects, as if they were reading it to a child or reading it in dramatic style. Beside the comparison in continues speech style within and between speakers, emotional/theater speech is useful for acted emotion recognition.

7) Re-telling the Story: Participants are asked to re-tell the previous story in their own words, such task providing a spontaneous, continuous-speech style. Also in this task, speakers are also asked few questions about the story and their opinion, which gives a variety of spontaneous speech.

8) Interview: In this component participants were asked to talk about a topic that interests them, which is followed by a 
conversation about the topic they chose. The aim of this task is to capture spontaneous, engaged, narrative talk in informal, 'vernacular', speech style. Even though this speech style is hard to capture in formal interviews, a skilful empathetic interviewer could elicit such a speech style.

9) Map Task: In this task, two participants are involved in a game style task, where one participant is trying to guide the second through a map. The second speaker should draw a route in their map based on the instruction given by the first speaker. The maps are not identical and a few landmarks differ, which elicits spontaneous interactive speech. After completing the task, both speakers are asked to have a conversation about the task and the reasons for any confusion. Such speech style (both the instructional and conversational) is rich in phonological segments of truly spontaneous speech, which could be compared with formal speech.

10) Yes/No Elicitation: In this task the speakers is asked simple yes/no questions, to answer in any variation of 'Yes' and 'No'. Beside forensic applications, this task is useful for automatic phone-booking systems to cover most variations of yes/no answers.

\section{H. Annotations}

Recorded data are currently under annotation by the annotation team at the AusTalk center at Macquarie University in Sydney, the annotation including scripting and labeling the speech data. The start and the end of each component is set by the recording software. However, the actual speech start and end time have to be labeled as well. Even though the read speech has a known script, mistakes, repetition, or uncompleted items have to be checked and scripted. Obviously, given that it has no script, spontaneous speech has to be scripted and labeled from scratch. The annotation of what is said will be aligned at the phrase or sentence level, as well as a more detailed annotation at the phone level. All annotations will be available in the server to facilitate researchers access.

\section{Personnel}

Most of the speech scientists involved in planning and designing AusTalk were assigned to manage the recording progress in their site. Their insight and full understanding of the project requirement helped in directing, supporting, and coordinating the data collection. A programming and engineering team was also available to build the AusTalk software and help fixing urgent problems. Research assistants were centrally trained to ensure a standard recording procedure for every site.

\section{INTERESTED ORGANIZATIONS AND SUPPORT}

The AusTalk project and its research outcomes are beneficial economically and educationally as well as politically. Several business companies, such as security business, as well as companies who are interested to automating their customer services (e.g. phone booking or calling centers) could use the AusTalk database to improve their systems to accommodate their Australian English speaking clients. Universities and research centers will benefit from this large database to diverse and scale-up their research findings. The AusTalk project provides a diverse and rich database to build a background model that will improve forensics and speaker identification results, which could have legitimate value in courts. Governments also could use such rich databases to support the identification of international criminals.

AusTalk is supported by several relevant associations: (1) the Australasian Speech Science and Technology Association (ASSTA) which is concerned with advanced understanding of the national spoken language as well as forensic speech science, and (2) the Human Communication Science Network (HCSNet) which created a network of speech scientists and also spawned the Thinking Head project [7]. The AusTalk database will be applicable to improve the Thinking Head project for Australian English speech.

\section{Difficulties FACED}

As for any big project, difficulties were faced from several aspect of the AusTalk design. Starting from suitable recording environment availability, to hardware and software failures.

The recording room in some sites had audible noise (e.g. loud air-conditioning, maintenance activity around the room, usage of loud devices), which made some rooms unsuitable for recording. In some cases alternative rooms were not available. Therefore, adjusting volume levels, as well as the 1 minute silent recording were required at each site to normalize the speech files against the silent recordings. However, those solutions are still not suitable for sudden and isolated noises, where the research assistant is asked to pause the recording in such incidents.

In the early stages of designing the Black Box, synchronizing the microphones audio signals with the camera video signals posed data synchronization problems, which were fixed by adding a 'sync' cable [16]. Even though the Black Box is designed in replicable way by having off-the-shelf components, replacements had to be sent from the AusTalk center to any of the regional site. Spare parts such as extra lights were shipped with the Black Box. Besides, research assistants were trained to use the equipment carefully to reduce such incidents.

As with any software, failures are expected, AusTalk had faced such failures, starting from failing to save files, ending with static noise being recorded. In the AusTalk center, a team of engineers and programmers were assigned to maintain and update the software for any errors and feedback they got from the research assistants. Also remote controlling the Black Boxes was done for urgent fixes.

Moreover, given the large data size (1000 speakers $\times 3$ hours of Audio-video recordings), storing the recorded sessions filled up the hard-disk capacities in a few days. Therefore, compression was necessary, which was performed on the video data only [16].

One of the AusTalk goals is to have the database available to research centers. Even though the consent forms have covered this issue, an ongoing argument was introduced about how much information to share. Especially the demographic questionnaire information, which could potentially violate participants' privacy. That is, based on the demographic questionnaire, a speaker could be identified. In the same time, such information is essential to study and associate the influence of the speaker history and their current accent and speech 
production. Therefore, limiting the access to such information to authorised researchers would reduce the risk of violating participants' privacy.

On the other hand, human errors are inevitable but could be reduced. Therefore, the research assistants centralized training sessions meant to reduce such errors. Also, research assistants were required to send a dummy recording to the AusTalk center, for quality checking on general recording, volume and light levels. Once this session is approved, the research assistant will be given the green light to start with real recordings. However; few other isolated human errors occurred, such as forgetting to check recording setting such as light level, or sound volume.

\section{FutURe Phases}

In the first phase of AusTalk protocol, few components were restricted to few recording sites for testing and validating their purposes and usability.

For example, emotion eliciting tasks were recorded only in one site. Future planing for eliciting emotions in speech and facial expression could be beneficial for improving understanding clients' emotions in phone-booking systems. For example, knowing if the calling client is annoyed in the automated-answering phone system is essential to transfer them to human employee, therefore reducing the risks of losing that client. Also associating facial with speech expression would be beneficial for psychological studies as well as for HCI applications.

Another restrained component was Australian languages versus Australian English, where indigenous Australian languages has been recorded in two site with limited numbers in the current phase. Therefore, the next phase of AusTalk will consider covering more Australian languages.

Another compromised example is having accented participants, that is; recording non-native speakers to capture association between mother language and pronouncing Australian English, which will be useful for linguistics and forensics research. Given the success achieved, the next stage for AusTalk is to expand the data collection for non-native speakers. Such collection have many potentials, including the governments collaboration against terrorist threats, by identifying them and their ethno-cultural group. In the new phase, a new component might be added asking the speakers to talk about a topic or translate the story in their own mother language(s). This component will capture the speakers' speech style and phonetics of their mother language for further comparisons.

In the long term, a decade or two later a follow up recording could be beneficial for further investigation on language changes, and also to cover future technologies' needs.

\section{Localization of Speech Data Collection}

The huge efforts and the careful planning by Australian speech scientists were behind the success of the AusTalk data collection. Having diverse components that accommodate most speech research areas, made this project a rich environment. Therefore, it is advantageous to use a similar but customized framework in any country which is interested in such research areas or preserving their local and regional accents and languages. Customization of each part of AusTalk design should be considered to accommodate the country's cultures and needs.

Besides being involved in speech research, every country might have different motivations. Some countries with diverse cultures, dialogues and languages could be motivated to collect those for preservation before those dialogues dies out with civilization. Also, countries which are threatened, for example, by terrorist attacks might be interested to collaborate for a world wide database to identify such criminals.

Even though the recording should cover most of majority and minority groups, languages and accents, depending on the country's culture and acceptance, the participant inclusion and exclusion criteria might differ. For example, countries who do not accept female video recording could implement few solutions around it. Instead of excluding female participants altogether, digitally covering face parts in the video, automatic feature extraction and storing instead of storing the raw video, or exclude video recording could solve this issue.

Some aspects should be taken into account regarding recording environments. On one hand, avoiding surrounding environmental noise while selecting recording times or rooms. For example, avoiding peak traffic hours, or avoid being close to construction sites, airports, train stations or even cities known to have high noise level. If the recordings would take place in the field, weather should be considered. Rainy or windy areas might need more time waiting for the weather to clear out, not only for the noise level but also for hardware protection. Extreme hot weather might need cooling equipment to avoid hardware failures. Also for extreme cold weather, cables and internal hardware components specifications should be planned.

While recording in regional or rural areas, hardware portability should be considered, starting from spare parts to power consumption. Given the heavy weight $(80 \mathrm{~kg})$, moving and carrying the Black Box in rural areas with unsealed roads should be considered for the safety of the hardware and feasibility of recording. Spare parts such as extra lights, harddrives, batteries and microphones should be included with the Black Box, as well as any required maintenance tools. If the recordings would take place in the field, where electricity is limited, appropriate portable rechargeable batteries or power generators should accompany the Black Box.

Storing sessions should be considered while recording in regional and different geographical locations, because running out of capacity will delay the recording process. For example, if Internet connection is limited or not available, or if mailing services are limited or not covered in those areas, estimated capacity should be calculated to count how many spare harddrives should be distributed with the Black Box.

The designed protocol for AusTalk covers most research areas, however, content obviously will be changed to support the countrys language needs. Not only on the phonetics side, but also to choose words, sentences, story and interview topics that are socially accepted. In the customized protocol, one could add few components, such as talking about a topic or translate the story in any other language(s) they could speak. Such component will give rich phonetics variation and speech style variability. 
Research assistant selection could be influenced by the geographical and cultural location. For example, if the session to be recorded in rural isolated place, research assistants might need to be trained on few more skills such as technical skills for immediate software or hardware fixes. Cultures who do not accept talking or sharing information with foreigners, would require training local personnel for recording.

\section{CONCLUSION}

A comprehensive speech database that covers theoretical and technical research areas has been designed by 30 Australian speech scientists, given the need prompted by the fast development of speech science. This database, named "AusTalk", was recorded in 17 sites in Australia, collecting 1000 diverse Australian English speakers, 3 one-hour sessions each at least one week apart. Reducing variability in content and in recording environment by standardizing procedure and recording equipment were the most important key issues in this database. In this paper, we described the framework used in "AusTalk" that could be exemplary for any multidisciplinary speech data collection. Problems faced, lessons learned and future phases of the project process are explained. The paradigm and design of this database is an ideal framework for any speech data collection. Therefore, we suggest the localization of this protocol and the potential customization depending on a country's specifications. Collecting such a speech database including accented groups is encouraged to boost speech research in many areas, including linguistics, speech and speaker recognition and forensics.

\section{ACKNOWLEDGMENT}

The AusTalk project was funded by the Australian Research Council (LIEF grant LE100100211) and was developed with support from the HCSNet research network and the Australasian Speech Science and Technology Association (ASSTA). The authors would also like to acknowledge the efforts and time, the speech scientists made available, which led to the success of the AusTalk data collection [18].

\section{REFERENCES}

[1] T. Polzehl, A. Schmitt, F. Metze, and M. Wagner, "Anger recognition in speech using acoustic and linguistic cues," Speech Communication, vol. 53, no. 9, pp. 1198-1209, 2011.

[2] S. Alghowinem, R. Goecke, M. Wagner, J. Epps, M. Breakspear, G. Parker et al., "From joyous to clinically depressed: Mood detection using spontaneous speech," in Twenty-Fifth International FLAIRS Conference, 2012.

[3] M. Little, P. McSharry, S. Roberts, D. Costello, I. Moroz et al., "Exploiting nonlinear recurrence and fractal scaling properties for voice disorder detection," BioMedical Engineering OnLine, vol. 6, no. 1, p. 23, 2007.

[4] S. McLeod and D. McKinnon, "Prevalence of communication disorders compared with other learning needs in 14500 primary and secondary school students," International journal of language \& communication disorders, vol. 42, no. S1, pp. 37-59, 2007.

[5] J. Ang, R. Dhillon, A. Krupski, E. Shriberg, and A. Stolcke, "Prosodybased automatic detection of annoyance and frustration in humancomputer dialog," in Proc. ICSLP, vol. 3. Citeseer, 2002, pp. 20372040 .

[6] L. Vidrascu and L. Devillers, "Five emotion classes detection in realworld call center data: the use of various types of paralinguistic features," Paraling2007, 2007.
[7] D. Burnham, R. Dale, K. Stevens, D. Powers, C. Davis, J. Buchholz, K. Kuratate, J. Kim, G. Paine, C. Kitamura et al., "From talking heads to thinking heads: A research platform for human communication science," ARC/NH\&MRC Special Initiatives, TS0669874, vol. 2011, 2006.

[8] M. Benzeghiba, R. De Mori, O. Deroo, S. Dupont, T. Erbes, D. Jouvet, L. Fissore, P. Laface, A. Mertins, C. Ris et al., "Automatic speech recognition and speech variability: A review," Speech Communication, vol. 49, no. 10, pp. 763-786, 2007.

[9] D. Reynolds, T. Quatieri, and R. Dunn, "Speaker verification using adapted gaussian mixture models," Digital signal processing, vol. 10, no. 1, pp. 19-41, 2000.

[10] J. Millar, P. Dermody, M. Harrington, and J. Vonwiller, "A national database of spoken language: concept, design, and implementation," in First International Conference on Spoken Language Processing, 1990.

[11] cat. no. 2071.0. (2013) Cultural diversity in australia @Australian Bureau of Statistics. [Online]. Available: http://www.abs.gov.au/ausstats/abs@.nsf/Lookup/2071.0main+features 902012-2013

[12] M. Walsh, "Overview of indigenous languages of australia," Romaine ed, pp. 27-48, 1991.

[13] P. McConvell and N. Thieberger, "State of indigenous languages in australia2001," Environment Australia. Canberra: Department of the Environment and Heritage, 2001.

[14] T. Wykes, "Can the psychiatrist learn from the psycholinguist? detecting coherence in the disordered speech of manics and schizophrenics," Psychological Medicine, vol. 11, no. 03, pp. 641-642, 1981.

[15] A. Postma, "Detection of errors during speech production: A review of speech monitoring models," Cognition, vol. 77, no. 2, pp. 97-132, 2000.

[16] D. Burnham, D. Estival, S. Fazio, J. Viethen, F. Cox, R. Dale, S. Cassidy, J. Epps, R. Togneri, M. Wagner et al., "Building an audio-visual corpus of australian english: large corpus collection with an economical portable and replicable black box," in Twelfth Annual Conference of the International Speech Communication Association, 2011.

[17] M. Wagner, D. Tran, R. Togneri, P. Rose, D. Powers, M. Onslow, D. Loakes, T. Lewis, T. Kuratate, Y. Kinoshita et al., "The big australian speech corpus (the big asc)," in 13th Australasian International Conference on Speech Science and Technology, 2010, pp. 166-170.

[18] D. Burnham, E. Ambikairajah, J. Arciuli, M. Bennamoun, C. Best, S. Bird, A. Butcher, C. Cassidy, G. Chetty, F. Cox et al., "A blueprint for a comprehensive australian english auditory-visual speech corpus," in Selected Proceedings of the 2008 HCSNet Workshop on Designing the Australian National Corpus, ed. Michael Haugh et al, 2009, pp. 96-107. 